la cytosine arabinoside à faible dose (leucémies aiguës myéloblastiques), les rétinoïdes (leucémies aiguës promyélocytaires), les dérivés de la vitamine $\mathrm{D}_{3}$ (fibroses malignes), l'interféron (leucémies à tricholeucocytes), le butyrate.

Quelle pourrait en être l'action? Ces produits agiraient-ils parce qu'ils modifient le promoteur, parce qu'ils agissent sur un activateur, ou, au contraire, sur un gène suppresseur? Ont-ils une action contre les facteurs de croissance par compétition sur le récepteur (comme l'interaction entre les téléocidines et le facteur de croissance épidermique)? Peuvent-ils changer l'épissage des ARN messagers et donc former un produit non dangereux?

Laurent Degos

Maître. de conférences agrégé en hématologie clinique.

Directeur de l'Unité de recherches d'immunogénétique de la transplantation humaine (Inserm U93). Hôpital Saint-Louis, 2, place du Dr-A.-Fournier, 75475 Paris Cedex 10.

\section{Les cultures de cellules leucémiques}

La transformation d'une cellule normale en une cellule maligne implique une série de modifications, dont des modifications chromosomiques (mutationnelles ou épigénétiques). La cellule maligne, immortalisée grâce à un renouvellement autonome, se bloque à un stade précoce de différenciation, au cours duquel la cellule acquiert des spécificités propres à son expression phénotypique et à sa fonction. Le fait que certaines tumeurs et leucémies murines et humaines peuvent être amenées à se différencier, permet d'évoquer la possibilité que la cellule maligne n'a pas complètement perdu les gènes régulateurs de la pousse et de la différenciation normale, et peut, dans certaines conditions, redevenir normale.

La reversion de la malignité a été obtenue par ségrégation chromosomique et par échanges chromosomiques après hybridation entre différents types cellulaires. Mais la reversion à un phénotype non malin a aussi été possible en l'absence d'échanges chromosomiques. En effet, la culture de progéniteurs de blastes en milieu semi-solide a montré que certaines cellules de colonies leucémiques pouvaient se différencier et garder les modifications chromosomiques de la leucémie aiguë initiale (ce marqueur devenant par la suite indispensable pour vérifier l'origine clônale leucémique des cellules différenciées de la descendance). Les modifications chromosomiques responsables de la transformation maligne impliqueraient donc des modifications au niveau de gènes autres que ceux responsables de l'induction de la différenciation.

Dans la cellule maligne, les gènes qui maintiennent l'équilibre entre prolifération et différenciation demeurent accessibles mais non fonctionnels. L'existence de lignées cellulaires immortelles de phénotype normal montre que les fonctions de prolifération et de différenciation sont génétiquement séparées. Ces gènes doivent néanmoins garder des liens puisqu'il est actuellement habituel d'observer une différenciation cellulaire concomittante à un arrêt de prolifération. La cellule normale ou maligne, sous l'action de facteurs exogènes, donne naissance à une famille de cellules complètement différenciées à potentiel mitotique faible, vouées à la destruction. L'arrêt de la prolifération est alors secondaire au processus de différenciation. Des études in vitro montrent cependant que la provocation d'un arrêt de prolifération des cellules malignes permet, en rétablissant l'équilibre normal prolifération/différenciation, de déclencher la différenciation cellulaire.

L'intérêt récent porté aux oncogènes cherche à identifier les gènes responsables de l'immortalisation et de la maturation anormale des cellules malignes. L'expression généti- que de certains oncogenes est augmentée dans les lignées malignes. Ce fait, associé aux découvertes liant les produits protéiques de certains de ces gènes à des récepteurs membranaires de facteurs de croissance, semble confirmer l'hypothèse de Sachs [I], à savoir que l'origine du processus malin pourrait être dû au passage d'un état induit à un état constitutif de l'expression des gènes qui contrôlent la prolifération cellulaire. L'étude des cellules leucémiques après différenciation cellulaire, montre en outre que l'état différencié est associé à une diminution de l'amplification de ces oncogènes (cmyc, N-ras) dans les neuroblastomes et dans les lignées promyélocytaires humaines.

Le processus malin apparaît donc réversible in vitro avec l'apparition de cellules mures, fonctionnellement compétentes. Les agents induisant la différenciation de cellules malignes sont maintenant nombreux, mais leur mécanisme d'action peu connu. Il demeure en effet difficile à l'heure actuelle de déterminer si les modifications observées au cours du processus de différenciation sont liées à l'action de la drogue ou au processus de différenciation lui-même. Paradoxalement, beaucoup d'inducteurs de la différenciation sont des agents connus comme induisant des tumeurs (les phorbol di-esters) ou comme des cancérigènes ou mutagènes, dont la plupart des drogues antinéoplasiques (5-azacytidine, bromodeoxyurïdine, méthotrexate, actinomycine $\mathrm{D}$, cytosine arabinoside...). Peut-on en conclure que leur action en tant que cancérigène est applicable à leur effet différenciateur?

On se souviendra néanmoins que l'analogue de base cytidine, la 5-azacytidine, entraîne une hypométhylation quand elle est incorporée au niveau de l'ADN et provoque ainsi l'expression de gènes de différenciation. Des lésions nucléiques, proches de celles responsables de traits cancérigènes ou mutationnels, peuvent être responsables de mutations ou de modifications de l'expression de gènes régulateurs de la prolifération et/ou de la différenciation cellulaire. D'autres agents, 


\section{REFERENCES}

2. Collins S. J., Gallo R., Gallagher R. Continuous growth and differentiation of human mycloid leukemic cells in suspension culture. Nature 1977; 270 : 347-9.

3. Dalla Favera R., Wong-Staal F., Gallo $R$ Oncogene amplification in promyelocytic leukemia cell line HL-6o and primary leukaemic cells of the same patient. Nature 1982; 299:61-3.

4. Murray M., Cunningham J., Parada L., Dautry F., Lebowitz P., Weinberg R. The HL-6o transforming sequence: $A$ ras oncogene coexisting with altered myc genes in hematopoietic tumors. Cell 1983; 33: 749-57.

5. Colbert D. A., Fontana J. A., Bode U., Deisseroth $A$. B. Changes in the translational activity of polyadenylated messenger RNA of HL-6o promyclocytic leukemia cells associated with myeloid or macrophage differentiation. Cancer Res. 1983; 43 : 229-34.

6. Westin E., Wong-Staal F., Gelmann E. et al. Expression of cellular homologues of retroviral oncogenes in human hematopoietic cells. PNAS 1982; $79: 2490-4$.

7. Marx J. L. New ways to "mutate" genes. Science 1984; 225:819.

8. Izant J. G., Weintraub H. Inhibition of thymidine kinase gene expression by anti-sense RNA a molccular approach to genetic analysis. Cell $1984 ; 36$ : $1007-15$. physiologiques, peuvent être aussi inducteurs : les dérivés de la vitamine $A$ (les acides rétinoïques trans et cis), la vitamine $\mathrm{D}_{3}$ sous sa forme activée : 1-25 $\mathrm{OH}$, le butyrate de sodium ...

Mais la reversion de la malignité ne dépend pas seulement de l'action de différents agents. Plusieurs faits tendent à prouver que le problème est plus complexe : toutes les cellules malignes ne peuvent être induites à se différencier, soit parce que les marqueurs actuels d'évaluation du stade de différenciation sont maigres, soit par défaut d'agents efficaces; les agents différenciants sont actifs sur certaines lignées leucémiques et non sur d'autres, et spécifiques d'une voie particulière de différenciation hématopoiétique; la régression de la malignité sous l'influence des agents inducteurs de différenciation dépend du temps d'incubation en présence de l'agent : le point de non retour peut être obtenu en 24-48 heures ou nécessite la présence constante de la drogue dans le milieu; quel que soit l'agent différenciant, il persiste souvent un faible pourcentage de cellules non différenciées, soulignant une résistance spécifique de certaines cellules malignes à devenir normales, ainsi que l'hétérogénéité des populations cellulaires malignes. Certaines cellules malignes sont donc capables d'aller jusqu'à une différenciation terminale, mais la persistance d'anomalies chromosomiques, ainsi que la possibilité, in vitro, de retourner à l'état malin, tendent à confirmer que la cellule différenciée n'est que phénotypiquement normale.

\section{Christine Chomienne}

Assistante des hôpitaux. Hôpital Saint-Louis.

Jean-Pierre Abita

Directeur de l'Unité de recherches de cinétique des populations cellulaires en hématologie et cancérologie (Inserm U 204). Hôpital Saint-Louis.

Maître de recherches au Cnrs.

\section{Le point de vue de la biologie moléculaire}

Au cours des dernières années, la toute récente biologie moléculaire du cancer a connu deux générations de concepts. Tout d'abord celle de l'oncogène cellulaire, c'est-à-dire d'un gène normalement présent dans les cellules qui, à la suite de modifications, peut jouer un rôle central dans la tumorigenèse. Puis celle de la coopération entre oncogènes, donc de la nécessité de modifier plusieurs gènes pour aboutir à une cellule tumorale.

Dans le cadre d'une éventuelle application clinique des travaux sur les oncogènes, la question centrale devient donc : sera-t-il suffisant d'agir au niveau d'un seul oncogène, ou, seuls des mécanismes pléiotropiques permettent-ils de normaliser une cellule tumorale?

L'exemple le plus classique d'un arrêt de prolifération pour une cellule tumorale est fourni par la différenciation cellulaire. En effet, la perte de la capacité de prolifération au cours de la différenciation cellulaire est un fait solidement établi, notamment pour la moelle osseuse. Pour la plupart des tissus cancéreux, la relation entre prolifération et différenciation se traduit par la double constatation d'une prolifération accrue et d'un arrêt de la différenciation normale, ces deux caractères étant diversement associés selon le type de cancer. Il est remarquable que pour des lignées tumorales établies au laboratoire, certaines substances soient capables d'induire une différenciation des cellules cancéreuses avec arrêt de leur prolifération. La différenciation cellulaire modifiant l'expression de très nombreux gènes, on peut se demander si l'arrêt de la prolifération nécessite la modification de l'expression de tous les oncogènes activés dans la cellule tumorale. Le système modèle le plus étudié est la lignée cellulaire HL .60 établie à partir d'une leucémie aiguë promyélocytaire humaine [2]. Il a 